\title{
A REMARK ON C-COMPACT SPACES
}

\author{
BHAMINI M. P. NAYAR \\ (Received 13 March 1997) \\ Communicated by J. A. Hillman
}

\begin{abstract}
It has been observed by a number of researchers that although it is well-known that all continuous functions defined on $\mathrm{C}$-compact spaces are closed functions, this property does not characterize C-compact spaces. In this note we employ the notion of strongly subclosed relations to prove that a space is $\mathrm{C}$-compact if and only if all functions on it with strongly subclosed inverses are closed functions.
\end{abstract}

1991 Mathematics subject classification (Amer. Math. Soc.): primary 54D25; secondary 54C99. Keywords and phrases: C-compact spaces, strongly subclosed relations, closed functions.

Throughout this note all spaces are Hausdorff spaces. Let $X$ be a space and let $A \subset X$. We denote the closure of $A$ by $\bar{A}$ and the collection of open sets which contain $A$ by $\Sigma(A)(\Sigma(x)$ if $A=\{x\})$; we use the notation $\Gamma(x)=\{V-\{x\}: V \in \Sigma(x)\}$. The $\theta$-closure of $A$, denoted by $c_{\theta} A$, is $\bigcap_{\Sigma(A)} \bar{V}$ and the $\theta$-adherence of a filterbase $\Omega$, denoted by $a d_{\theta} \Omega$, is $\bigcap_{\Omega} c l_{\theta} F$. These notions were introduced by Veličko for the purpose of studying $\mathrm{H}$-closed spaces and have subsequently received wide usage (see [1, 2]). A relation $F \subset X \times Y$ is strongly subclosed if $\operatorname{ad}_{\theta} F(\Gamma(x)) \subset F(x)$ for each $x \in X$ for which $\Gamma(x)$ is a filterbase on $X$ [1]. We will say that a function $g: X \rightarrow Y$ has a strongly subclosed inverse if the relation $g^{-1}$ is strongly subclosed. It is not difficult to prove that continuous, and indeed $\theta$-continuous [1], functions have strongly subclosed inverses.

A space $X$ is said to be $C$-compact if for each closed $A \subset X$, each cover of $A$ by open subsets of $X$ contains a finite subfamily $\mathscr{V}$ such that $\{\bar{V}: V \in \mathscr{V}\}$ covers $A$. A space is $\mathrm{H}$-closed if it is a closed subspace of every space in which it is embedded. It is known that a space $X$ is $C$-compact if and only if each closed $A \subset X$ and filterbase $\Omega$ on $A$ satisfy $A \cap a d_{\theta} \Omega \neq \emptyset$ and that a space $X$ is H-closed if and only if every filterbase on $X$ has nonempty $\theta$-adherence [3]. We are now in a position to give a

(C) 1998 Australian Mathematical Society $0263-6115 / 98 \$ A 2.00+0.00$ 
proof of the theorem.

THEOREM. A space $X$ is $C$-compact if and only if all functions on $X$ with strongly subclosed inverses are closed functions.

ProOF. Necessity. Let $A \subset X$ be closed, and $g: X \rightarrow Y$ have a strongly subclosed inverse. If $y$ is a limit point of $g(A)$ then $\Omega=\left\{g^{-1}(W) \cap A: W \in \Gamma(y)\right\}$ is a filterbase on $A$ and hence $\emptyset \neq A \cap a d_{\theta}(\Omega) \subset A \cap g^{-1}(y)$. So $g(A)$ is closed.

Sufficiency. Suppose $A$ is a closed subset of $X$ and that $\Omega$ is a filterbase on $A$ such that $A \cap a d_{\theta} \Omega=\emptyset$. Since continuous functions have strongly subclosed inverses, it follows that $X$ is H-closed and hence that $A \neq X$. Choose $v \in A$ and define $g: X \rightarrow Y$ by $g(x)=x$ if $x \in A, g(x)=v$ if $x \in X-A$, where $Y=X$ with the topology $\{V \subset X: v \in X-V$ or some $F \in \Omega$ satisfies $F \subset V\}$. Then $Y$ is Hausdorff and $g^{-1} \subset g(X) \times X$ is strongly subclosed since $\Gamma(y)$ is a filterbase on $Y$ only if $v=y$, and $a d_{\theta} g^{-1}(\Gamma(v)) \subset a d_{\theta} \Omega \subset X-A \subset g^{-1}(v)$. There is an $F_{0} \in \Omega$ and $W \in \Sigma(v)$ with $\bar{W} \cap F_{0}=\emptyset$ in $X$. It follows that $g\left(F_{0}\right)=F_{0} \subset A-W=g(A-W)$, so $v \in \overline{A-W}-(A-W)$ in $Y$. Since $A-W$ is closed in $X$ the function $g$ is not a closed function.

\section{References}

[1] J. E. Joseph, 'Multifunctions and graphs', Pacific J. Math 79 (1978), 509-529.

[2] R. L. Krystock, 'Adherent compact spaces', Proc. Amer. Math. Soc. 107 (1989), 1117-1125.

[3] N. V. Veličko, 'H-closed topological spaces', Mat. Sb. 70 (1966), 98-112; English transl. in Amer. Math. Soc. Transl. 78 (1968), 102-118.

Department of Mathematics

Morgan State University

Baltimore, MD 21251 USA

e-mail: bnayar@morgan.edu 